\title{
New advances in monitoring and triggering of mechanical ventilation
}

\author{
SHI Yan ${ }^{1,2}$, ZHANG BoLun $^{1}$, WANG ZeDan ${ }^{3}$, CAI MaoLin ${ }^{1,2} \&$ SHEN DongKai ${ }^{1,2^{*}}$ \\ ${ }^{1}$ School of Automation Science and Electrical Engineering, Beihang University, Beijing 100191, China; \\ ${ }^{2}$ Beijing Engineering Research Center of Diagnosis and Treatment of Respiratory and Critical Care Medicine, Beijing Chaoyang Hospital, \\ Beijing 100043, China: \\ ${ }^{3}$ Qingdao Women \& Children Hospital, Qingdao 266000, China
}

Received July 27, 2016; accepted September 2, 2016; published online October 11, 2016

\begin{abstract}
Mechanical ventilation has been widely used in intensive care unit (ICU) to provide adequate air exchange to patients with respiratory difficulty. It helps patients with a ventilator that presses air into patients' lungs at triggering time. As a key therapy in ICU, mechanical ventilation is of great significance in clinical research of respiratory treatment. For example, simulation research of mechanical ventilation can be made through dynamics equations of pneumatic system [1-3]. With improvement of monitoring technique, monitoring and triggering of mechanical ventilation system have become focuses of research of respiratory treatment. Because of suddenness of respiratory diseases, respiratory parameters of patients need to be collected and presented timely and patient-ventilator synchrony of triggering mainly depends on which respiratory parameter is used to trigger mechanical ventilation.
\end{abstract}

For ventilators that are widely used in ICU at present, several parameters are monitored during mechanical ventilator. Flow and pressure are two most important parameters of patients with mechanical ventilation and main situation of patients can be reflected on by these two parameters. However, because flow and pressure of patients' lungs are difficult to be monitored directly, present ventilators mainly obtain data from airway. Considering air leakage of mechanical ventilation, traditional monitoring method can be not very reliable to reflect on states of patients, especially patients' need to breathe.

There are mainly two triggering modes for present ventilators: control mode and assist mode. In control mode, all the parameters of ventilators are set stable before mechani-

\footnotetext{
*Corresponding author (email: shendk@vip.sina.com)
}

cal ventilation and ventilators are triggered automatically during ventilation. In assist mode, triggering of ventilators is carried out by flow or pressure of patients. Clinical researches show that flow triggering has less delay triggering time and takes less work of breathing [4-6]. There are two main shortcomings of traditional triggering methods. First, because there is time interval between patients' urge to breathe and change of flow and pressure which can be illustrated by Figure 1. Second, parameters that are set stable before ventilation can hardly suit every patient, which damages patient-ventilator synchrony directly, and bad patient-ventilator synchrony leads to increase of sedatives and proportion of cut airway.

Neutrally adjusted ventilator assist (NAVA) is a new method to monitor and trigger mechanical ventilation system that basically overcomes most shortcomings of traditional monitoring and triggering methods. NAVA uses diaphragmatic electromyographic (EMGdi) signals to trigger ventilators. EMGdi is a weak biological signal generated by the diaphragmatic during respiration [7,8]. It appears earlier than the change of both airway pressure and flow. As can be seen in Figure 1, NAVA uses diaphragmatic contraction to start ventilation while traditional triggering uses change of pressure and volume, and as a result, NAVA has less delay time than traditional triggering. Normal delay time of flow and pressure triggering is around $80 \mathrm{~ms}$ while delay time of NAVA is around $16 \mathrm{~ms}[9,10]$. There are two ways to obtain EMGdi signals: one way is to put monitoring electrode onto skin of patients and obtain surface electromyographic (EMG) signals which are comprehensive results at time and space of skin surface from electrical activity of muscle under epidermis. The other way is to put monitoring electrode 


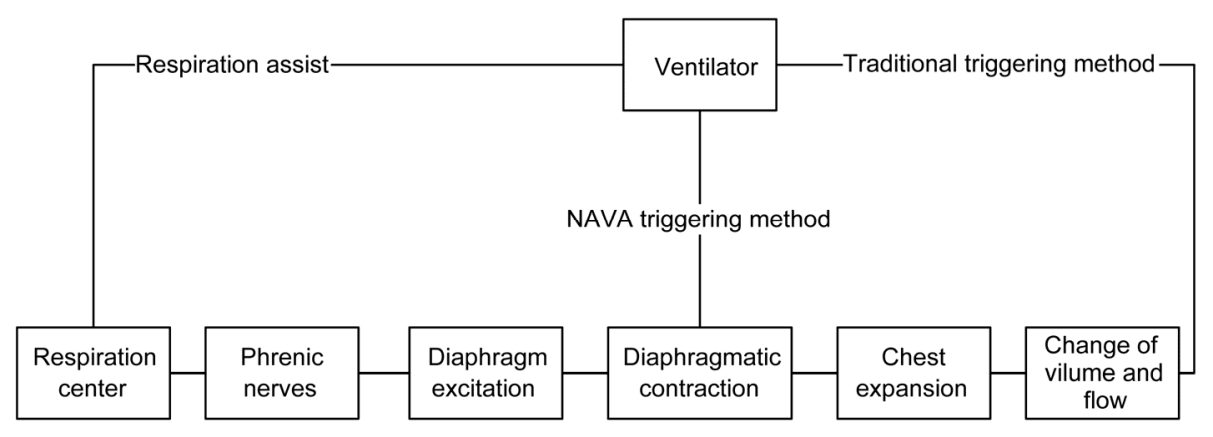

Figure 1 Comparison between NAVA and traditional triggering.

into stomach of patients through esophagus and obtain EMGdi directly.

To summarize, there are both advantages and disadvantages of adopting NAVA. Firstly, because EMGdi signal appears at respiratory nervous system, it's more representative to reflect on patients' real need to breathe. Secondly, less delay time of NAVA leads to better patient-ventilator synchrony. Thirdly, NAVA triggering costs less work of breathe. As for disadvantages, because EMGdi is hard to obtain in a noninvasive way, it's difficult to balance efficiency and safety of triggering when adopting NAVA.

1 Shi Y, Niu J L, Cao Z X, et al. Working characteristics of a new ventilator with automatic secretion clearance function. Sci China Tech Sci, 2015, 58: 1046-1052

2 Mei S W, Wang J J, Tian F, et al. Design and engineering implementation of non-supplementary fired compressed air energy storage system: TICC-500. Sci China Tech Sci, 2015, 58: 600-611

3 Wang Y S, Wang K, Lin F, et al. Performance analysis and im- provement of a high flow coefficient centrifugal compressor. Sci China Tech Sci, 2014, 57: 1647-1657

4 Rouby J J, Lu Q, Goldstein I. Selecting the right level of positive end-expiratory pressure in patients with acute respiratory distress syndrome. Am J Respir Crit Care Med, 2002, 165: 1182-1186

5 Tobin M J, Bouchard L. Respiratory Pressure Volume Curves in Tobin MJ Eds Principles and Practice of Intensive Care Monitoring. New York: McGram-Hill, 1998. 597-616

6 Hill N S, Levy M M. Ventilator Management Strategies for Critical Care. New York: MarcelDekker, 2001. 171-241

7 Zheng H W, Wang R B, Qiao L K, et al. The molecular dynamics of neural metabolism during the action potential. Sci China Tech Sci, 2014, 57: 857-863

8 Zhao S S, Pei W H, Zhao H, et al. A novel linear microprobe array for the fabrication of neural microelectrodes. Sci China Tech Sci, 2015, 58: 346-351

9 Lissens M A. Motor evoked potentials of the human diaphragm elicited through magnetic transcranial brain stimulation. J Neurol Sci 1994, 124: 204-207

10 Sinderby C, Navalesi P, Beck J, et al. Neural control of mechanical ventilation in respiratory failure. Nat Med, 1999, 5: 1433-1436 